\section{Psychological Medicine}

cambridge.org/psm

\section{Correspondence}

*Hui-Yuan Chen and Renin Chang contributed equally.

Cite this article: Chen $\mathrm{H}-\mathrm{Y}$, Chang $\mathrm{R}$, Wei JC-C (2022). Dementia in Scottish military veterans: early evidence from a retrospective cohort study. Psychological Medicine 1-1. https:// doi.org/10.1017/S0033291721005237

Received: 24 November 2021 Revised: 1 December 2021

Accepted: 2 December 2021

Author for correspondence: James Cheng-Chung Wei, E-mail: wei3228@gmail.com

\title{
Dementia in Scottish military veterans: early evidence from a retrospective cohort study
}

Hui-Yuan Chen ${ }^{1, \star}$, Renin Chang ${ }^{2, \star}(\mathbb{D})$ and James Cheng-Chung Wei ${ }^{1,3,4}$

${ }^{1}$ Institute of Medicine, Chung Shan Medical University, Taichung, Taiwan; ${ }^{2}$ Department of Emergency Medicine, Kaohsiung Veterans General Hospital, Kaohsiung, Taiwan; ${ }^{3}$ Department of Allergy, Immunology and Rheumatology, Chung Shan Medical University Hospital, Taichung, Taiwan and ${ }^{4}$ Graduate Institute of Integrated Medicine, China Medical University, Taichung, Taiwan

\section{To the Editor:}

We read with great interest the paper by Bergman, Mackay, and Pell (2021) about dementia in veterans and non-veterans. Authors found that there was no evidence in the subsequent risk of dementia between veterans and non-veterans (Bergman et al., 2021). This study has provided an excellent basis for a follow-up study of the association of veterans with dementia. However, there are some concerns we would like to discuss further.

First, we note that there's possibility of important residual confounders. We suggest further match or adjust known risk factors of dementia to rule out residual confounding bias, such as hypertension, diabetes, hypercholesterolemia, obesity, and cardiovascular diseases. Further subgroup analysis on these important covariates for clinical applications would be appreciated (Baumgart et al., 2015; Shang et al., 2020). Additionally, it would be necessary for further matching or stratified analysis of periodontal disease and chronic inflammatory diseases (Lee et al., 2016; Ma et al., 2021).

Second, we also concern about the coding accuracy of ICD-9 and -10 for dementia, posttraumatic stress disorder, and mood disorder that may be underdiagnosed in many claimbased databases. We suggest that the authors to provide validation data or references for the definition of outcome to avoid information bias.

We are grateful for the work done by the authors and looking forward to their response.

Conflict of interest. None declared.

\section{References}

Baumgart, M., Snyder, H. M., Carrillo, M. C., Fazio, S., Kim, H., \& Johns, H. (2015). Summary of the evidence on modifiable risk factors for cognitive decline and dementia: A population-based perspective. Alzheimer's \& Dementia, 11(6), 718-726. https://doi.org/10.1016/j.jalz.2015.05.016.

Bergman, B. P., Mackay, D. F., \& Pell, J. P. (2021). Dementia in Scottish military veterans: Early evidence from a retrospective cohort study. Psychological Medicine, 1-6. https://doi.org/10.1017/s0033291721002440.

Lee, Y.-T., Lee, H.-C., Hu, C.-J., Huang, L.-K., Chao, S.-P., Lin, C.-P., ... Chen, C.-C. (2016). Periodontitis as a modifiable risk factor for dementia: A nationwide population-based cohort study. Journal of the American Geriatrics Society, 65(2), 301-305. https://doi.org/10.1111/jgs.14449.

Ma, K. S., Hasturk, H., Carreras, I., Dedeoglu, A., Veeravalli, J. J., Huang, J. Y., ... Wei, J. C. (2021). Dementia and the Risk of Periodontitis: A Population-Based Cohort Study. J Dent Res, 13, 220345211037220. https://doi.org/ $10.1177 / 00220345211037220$.

Shang, Y., Fratiglioni, L., Marseglia, A., Plym, A., Welmer, A., Wang, H., ... Xu, W. (2020). Association of diabetes with stroke and post-stroke dementia: A population-based cohort study. Alzheimer's \& Dementia, 16(7), 1003-1012. https://doi.org/10.1002/alz.12101. 